\title{
Chromatin dynamics in regeneration epithelia: Lessons from Drosophila imaginal discs
}

\author{
Elena Vizcaya-Molina ${ }^{\mathrm{a}}$, Cecilia C. Klein ${ }^{\mathrm{a}, \mathrm{b}, \mathrm{c}}$, Florenci Serras ${ }^{\mathrm{a}}$, Montserrat Corominas ${ }^{\mathrm{a}, *}$ \\ ${ }^{a}$ Departament de Genètica, Microbiologia i Estadística, Facultat de Biologia and Institut de Biomedicina (IBUB), Universitat de Barcelona (UB), Barcelona 08028, \\ Catalonia, Spain \\ ${ }^{\mathrm{b}}$ Centre for Genomic Regulation (CRG), The Barcelona Institute of Science and Technology (BIST), Spain \\ ${ }^{\mathrm{c}}$ Universitat Pompeu Fabra (UPF), Barcelona 08003, Catalonia, Spain
}

\section{A R T I C L E I N F O}

\section{Keywords:}

Regeneration

Chromatin

Enhancer

Gene expression

Drosophila

\begin{abstract}
A B S T R A C T
During the process of regeneration, a switch in the transcription program occurs in cells that contribute to the reconstruction of the missing tissue. Early signals released upon damage are integrated into the chromatin of responding cells to change its activity and function. Changes in chromatin dynamics result in transcriptional reprogramming, this is the coordinated regulation of expression of a specific subset of genes required for the regeneration process. Here we summarize changes in gene expression and chromatin dynamics that occurs during the process of regeneration of Drosophila imaginal discs.
\end{abstract}

\section{Introduction}

Regeneration is the process by which organisms reconstruct the original shape, size and function of body parts that have been physically or functionally lost or damaged. Regeneration can denote both the continuous cellular self-renewal of particular structures or tissues (physiological regeneration or tissue homeostasis) and the restoration of damaged tissue or lost structures (reparative regeneration) (reviewed in [1]). In 1901 Thomas H. Morgan attempted to refine the concept of regeneration by coining the terms epimorphosis to refer to regenerative phenomena in which the formation of the new part involves cellular proliferation and morphallaxis to refer to those cases in which regeneration results from the remodeling of existing material without cellular proliferation [2]. Epimorphosis can be found, for instance, in zebrafish heart and fin regeneration [3] and Hydra is one example where morphallaxis takes place [4].

Regeneration can occur at multiple levels of biological organization throughout metazoans and, accordingly, a classification has been proposed to describe regeneration, ranging from single-cell to tissue, organ, structural and whole-body regeneration [5,6]. Moreover, as already suggested by Morgan [2], regenerative capacity seems to be regulated by a number of fundamental traits, such as age, body size, life stage or wound-healing response (reviewed in [7-9]).

The capacity to regenerate is not universal and varies greatly, not only from one species to another, but also between tissues and organs or between developmental stages of the same species (reviewed in
[5,10-12]). Planarians, for instance, can reconstruct their whole body from a tiny piece of almost any of their body parts; other Platyhelminthes, however, are unable to regenerate their heads and die after head amputation $[13,14]$. Such regenerative differences between closely related species do not only occur at high levels of biological organization, where patterning, development, and production of many different cell types occur; regeneration following less complex levels of biological organization, such as tissue regeneration, can also be similarly divergent. This, for example, is the case of differing skin regeneration between the mouse lab model (Mus musculus) and the African spiny mouse (Acomys). While the African spiny mouse can regenerate skin perfectly, the mouse laboratory model is unable to regenerate and instead forms fibrotic scars [15]. In addition, regeneration also depends on the developmental stage or maturation of the individual. In mammals, fetuses and newborn individuals have a relatively high degree of regenerative capacity, which is lost in the adult: newborn mice can heal their heart or skin better than adults [16,17]. In humans, distal phalanx regeneration after amputation has been observed in young children but not in adults (reviewed in [18]). To a certain extent, the same occurs in some insects: the capacity to regenerate specific organs that is observed at larval stages seems to be lost in the Drosophila adult (reviewed in [19,20]).

Injury is unavoidable for animals in the wild, where they can lose body parts to predators or due to other natural distresses. Thus, reparative regeneration involves the well-coordinated restoration of cells, tissues, and organs that have been physically or functionally lost. This

\footnotetext{
* Corresponding author.

E-mail address: mcorominas@ub.edu (M. Corominas).
} 
process must therefore recognize missing structures and recapitulate them, while simultaneously achieving functional integration between recently formed and preexisting tissues. Cells that contribute to the restoration process are guided to regions where they are needed; and once regeneration is complete, specific cues are required for signal termination. However, regeneration is not constrained to recovery of lost parts, which are rare and often caused by accidents, but also occurs in the renewal of cells that become damaged due to altered homeostatic conditions. On the other hand, in all instances, regeneration, whether reparative or physiological, stands as a widespread and advantageous trait of survival [21].

Different models for understanding regeneration, from invertebrates to mammals, are currently used and have provided considerable insight into the molecular mechanisms underlying regeneration, which seem to be remarkably conserved. A key question nowadays is how cells trigger nuclear reprogramming and transcriptional programs specific for regeneration. In this review, we explore the current understanding of changes in chromatin dynamics that contribute to the regenerative response of Drosophila imaginal discs. Imaginal discs are epithelial sacs that are the primordia of adult appendages and other cuticular structures. Imaginal discs can also serve as an example of reparative regeneration, since they are capable to regenerate after damage (reviewed in $[19,20,22,23])$. Damage can be induced physically, by microsurgery, or genetically, by genetic induction of cell death (reviewed in $[20,24])$. Whether discs undergo regeneration in the wild after cell death induced by cold or heat shocks, or other natural distresses, is currently unknown.

\section{Signaling to chromatin}

Damage, one way or another, activates regeneration programs in the living cells that proliferate to recover the injured area. These regeneration programs include several signaling pathways that are essential for normal development. Soon after an injury occurs, damaged cells release signals as reactive oxygen species (ROS), calcium waves or bioelectrical stimuli that are sensed as pro-regenerative signals by the adjacent living cells [25-31]. At the same time, injury causes inflammation, which results in the recruitment of immune cells to the wounded area. These immune cells release cytokines that are also sensed as pro-regenerative signals [32-37]. Altogether, these signals ultimately regulate the activation of signaling pathways such as, JNK and p38, WNT, Jak-STAT, EGFR/Ras/MAPK or Hippo [29,31,38-45]. Upon receiving such input, cells undergo extensive changes in chromatin activity and switch their transcriptional programs to rebuild the tissue that has been lost or injured (Fig. 1).

Chromatin plays a key role in determining the cellular fate and identity. Changes in chromatin dynamics underlie cell plasticity (the capacity of cells to take on characteristics of other cells) following injury $[46,47]$. The number and type of cells to be restored, as well as the source of new cells, may be different, however, in different species. Planarians, for example, use a population of stem cells called neoblasts that self-renew and also generate new cell types (reviewed in [48-50]). Among vertebrates, lens regeneration is basically restricted to some amphibians; whereas in frogs, the lens is regenerated by transdifferentiation of the cornea. Meanwhile, pigment epithelial cells of the newt dorsal iris can regenerate a new lens via transdifferentiation [51]. In other systems, such as Hydra, a combination of the two, stem cells and transdifferentiation processes, is required [52,53]. In the regenerating zebrafish heart, existing differentiated cardiomyocytes undergo dedifferentiation, reduce their contractile state and start cell division to generate new cardiomyocytes that replace lost heart mass [54,55]. The mammalian liver is also able to regenerate after injury in order to maintain proper homeostasis. This regeneration process consists of hepatocytes undergoing compensatory hyperplasia (an increase in cell number) as well as an increase in cell size (reviewed in [56]). Recent studies provide evidence that adult fly tissues such as flight muscles and the gut may also harbor quiescent stem cells, which can regenerate the tissue upon injury [57]. In the case of the wing disc, in addition to an increase in proliferation near the damaged tissue, there is reorientation of cell division and local respecification of vein and intervein fates [40,58,59].

Determining how signaling pathways integrate with chromatin dynamics during regeneration would provide a basis to understand the cell plasticity that is required to allow reconstitution of the missing tissue while disc identity is maintained [60]. During the course of regeneration, imaginal disc cells may also undergo transdetermination: a process whereby determined cells change their fate to that of a different disc identity [60-62]. In addition, transgression of compartment borders has been reported after massive damage in one compartment of the wing imaginal disc; the transgressing cells are genetically reprogrammed and acquire a new identity [63].

\section{Signaling integration}

The outcome of signaling is the transcriptional regulation of target genes that will elicit the final response. After entering the nucleus, the transmitted signals may modulate the activity of transcription factors that together with chromatin-remodelers and modifying enzymes and other epigenetic pathways, including non-coding RNAs (ncRNAs), will influence chromatin structure and activity in the cells contributing to the regeneration process (Fig. 1).

The fact that adult fruit flies cannot reset the transcriptional programs needed for regeneration could be explained by differences in the spatial and temporal regulation of gene expression, and may not be a consequence of the genes encoded in their genome. If regenerative signals are not properly sensed or integrated into the genome, then the

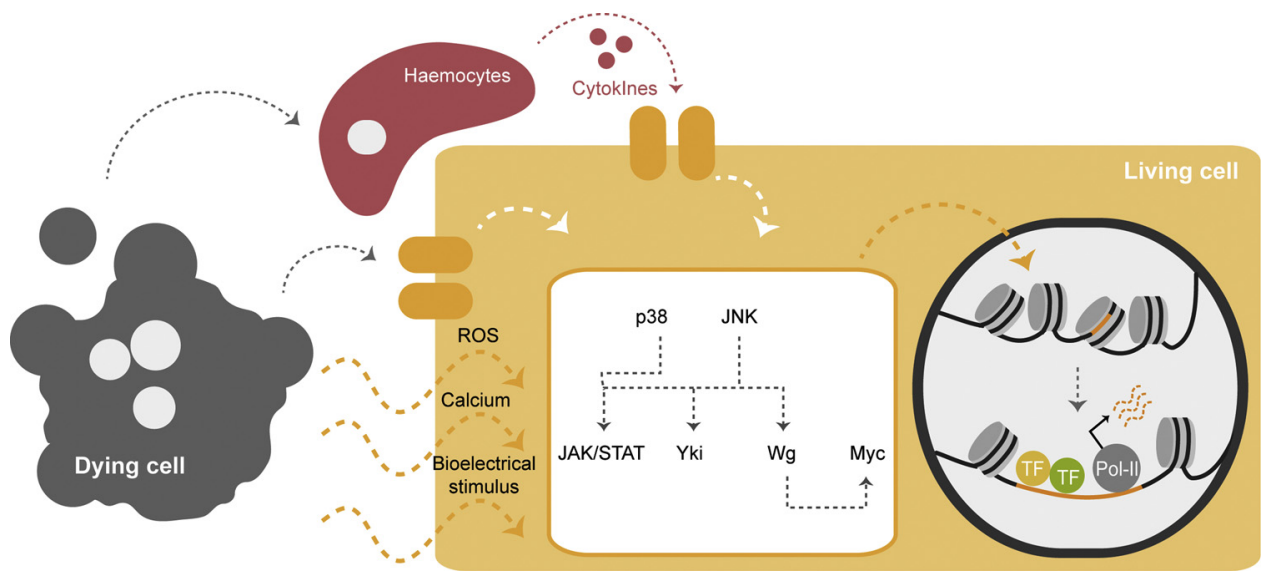

Fig. 1. Early regeneration signals. Depiction of how a living cell can sense different signals (ROS, calcium, bioelectrical stimuli, inflammatory signals and the release of different ligands). These signals are released from the damaged tissue (dying cell) to promote regeneration. As a consequence, several signaling pathways are activated. These are integrated into the nucleus to promote the transcription of regeneration genes. TF: transcription factor; Pol-II: RNA polymerase II. 
whole process fails. Indeed, it has previously been hypothesized that the chromatin landscape could determine the capacity for regeneration. Species that have acquired fully repressed states upon maturation would not allow the transcription of genes involved in regeneration. In contrast, animals that allow switches in chromatin states could more easily reprogram gene expression to cover the emerging needs of regeneration (reviewed in [64]).

\subsection{Polycomb and Trithorax groups in regeneration}

Historically, one of the most studied regulatory systems of chromatin-modifying factors in charge of the chromatin states involves the Polycomb group (PcG) and the Trithorax group (TrxG) of proteins, which act antagonistically to orchestrate the expression of key developmental genes. Originally discovered in Drosophila, PcG and TrxG are evolutionarily conserved proteins that play a role in cellular memory systems that maintain specific patterns of gene expression: repressed or active respectively (reviewed in [65]). Repressed states are best described by the presence of the repressive mark H3K27me3, meanwhile active states are depleted of it and enriched in other as H3K4me3 and H3K27ac. Proteins belonging to TrxG and PcG have been proven to be crucial to the process of regeneration by integrating signaling cues into the chromatin.

Studies in mouse skin epithelium have demonstrated that the depletion of epigenetic silencing mediated by PcG proteins helps to mediate upregulation of repair genes, after physical injury. Moreover, upregulation of H3K27 demethylases of the TrxG (Utx histone demethylase (UTX) and JmjC domain-containing protein 3 (JMJD3) is required in the blastema area to promote gene expression [66]. Similarly, studies in the fly indicate that transdifferentiation events in regeneration require an enhanced transcription state in which silencing is weakened by the coordinated action of the JNK pathway and PcG/TrxG members [67]. More recently it has been found that animals that are heterozygous for trithorax (trx) are unable to maintain activation of a developmental checkpoint that allows regeneration to occur. This defect is likely to be caused by abnormally high expression of puckered (puc), a negative regulator of Jun N-terminal kinase (JNK) signaling, at the wound site [68]. Additionally, it was discovered that the chromatin regulator Taranis (Tara), which belongs to the TrxG, stabilizes compartmental identities during the same transdifferentiation events [69]. In zebrafish fin regeneration, bivalent promoters containing H3K4me3 and H3K27me 3 histone modifications are converted to an active state by the action of an H3K27me3 demethylase [70]. Finally, it has been shown that separate modules of the WNT enhancer mediate damage response and age-dependent silencing in wing imaginal disc regeneration. PcG-mediated silencing of this enhancer limits the damage-responsive $w g$ expression in mature discs [71]. Altogether, signaling cues are sensed by the PcG and TrxG to allow the relaxed and active chromatin state in charge of the transcriptome of regeneration.

\subsection{The transcriptome of regeneration}

The regeneration transcriptome seems to be best described as the modulation of transcript levels of genes already expressed, rather than initiation of gene transcription de novo. For instance, in newt lens regeneration, RNA-seq results show that dorsal and ventral irides, which present different regeneration capacities, mostly differ in the number of transcripts, rather than their uniqueness [72]. In adult skeletal muscle, severe trauma induces transcriptional and post-transcriptional regulation of both coding and non-coding transcripts [73]. Moreover, transcriptomic studies in zebrafish and Drosophila have reported that such gene modulation is a burst of transcription that mainly occurs during the early regeneration stage and reverts over time [36,74,75]. In addition, by studying the transcriptome of regenerating wing imaginal discs, we found that there is global co-regulation of genes involved in regeneration; genes induced early on tend to be located close to one another in the linear genome. These clusters of upregulated genes are enriched in signaling pathway genes [75]. This observation suggests that co-regulation of gene expression could represent an efficient mechanism, as several clustered genes could be turned on at once by the same regulatory event. The fact that gene transcription is mostly explained by gene modulation indicates that regulatory elements, such as regeneration enhancers, could play a crucial role in the regulation of gene expression during the restoration process (reviewed in [76]).

\section{Enhancers as key regulatory elements in regeneration}

Enhancers are cis-regulatory DNA-regions that control gene expression. Some examples of specific damage-responsive enhancers have been reported in Drosophila and zebrafish in the context of regeneration. After damage, ectopic expression of WNT genes is found in early wing imaginal discs. This injury-induced expression is regulated by a bipartite enhancer at the WNT locus that is required for proper regeneration $[58,71,77]$. Transcriptomic analysis and epigenetic profiling have also revealed that the leptin $b$ gene (lepb) is strongly induced in regenerating hearts and fins of zebrafish; and a short DNA sequence element upstream and distal to lepb has been identified as a tissue regeneration enhancer element (TREE) [78]. By combining chromatin accessibility analysis with functional studies, the regulatory regions involved in whole-body regeneration have been recently characterized in Acoels [79].

Several properties of enhancers underlie their regulatory function. When bound to specific proteins, enhancers increase the level of transcription of an associated gene independently of the orientation and distance to its core promoter. Despite their position, enhancers can target their promoters through chromatin loops, which bring them in close spatial proximity [80-83]. It has been suggested that chromatin loops assemble an active chromatin hub, providing a more supportive environment for transcription than that created by transcription factors bound directly to their promoter alone. Indeed, many enhancer-promoter combinations usually share binding sites for common transcription factors, potentially leading to eRNA transcription (reviewed in $[84,85])$. The regulatory information provided by enhancers is encoded in short sequences that are recognized by transcription factors which bind to them and recruit cofactors, thus forming a complex that ultimately mediates activation of gene transcription [86-88] (Fig. 2A).

Many features that determine enhancer activity have been characterized; yet none of them seems to be a universal trait. Although active enhancers are located at accessible positions within the chromatin, enhancers per se are found in a default off state, determined by nucleosome positioning; they only become accessible under specific environmental conditions $[89,90]$. Chromatin accessibility is thus a key requirement for gene regulation and is one of the features that best predict enhancer activation [91].

When active, both, enhancers or promoters, are nucleosome depleted; however, the histones in the flanking nucleosomes often carry post-translational modifications, which provide a useful readout of enhancer activity. In active chromatin states, promoters are usually marked with H3K4me3, enhancers with H3K4me1, and both of them with H3K27ac [87,92-95]. Changes in H3K56ac seem an indicator of enhancer activation on the response to Notch signaling [96]. Furthermore, in silent chromatin states, promoters and enhancers are labeled with H3K27me3 (reviewed in [65,97]); while H3K9me3 is found in silent heterochromatin regions [98]. Thanks to the combinatorial action of histone marks, other chromatin states have been predicted. For instance, poised bivalent enhancers are those containing both H3K4me1 and H3K27me3 [99]; while latent enhancers are those that are not labeled with any type of mark, which means they can only be activated upon stimulation through signaling pathways [100].

Although histone modifications are one of the best predictors of enhancer activity, they present two major weak points. Usually, there is 


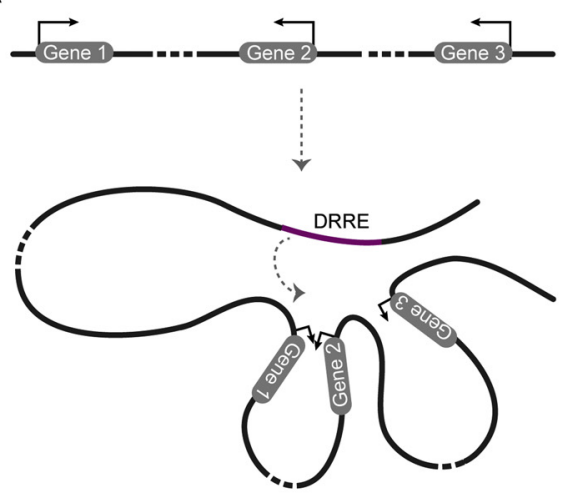

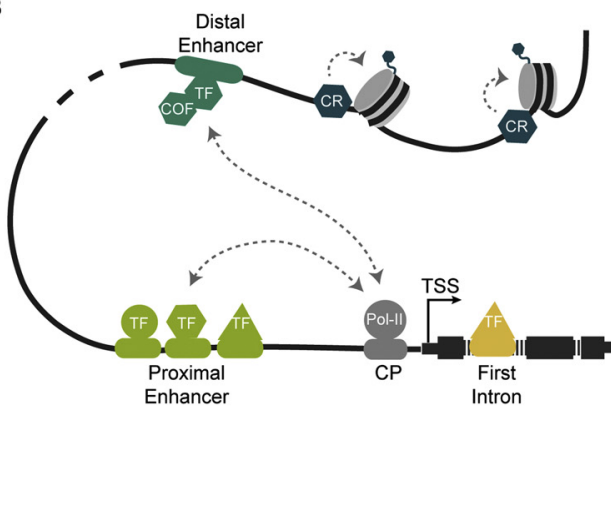

Fig. 2. Chromatin regeneration hubs. a) Illustration showing a putative chromatin regeneration hub. Spatial proximity generated by chromatin loops brings three genes, located at different genomic locations, into close contact, so they can be co-regulated by a single eDRRE; b) Illustration showing the three DRRE types and their accessibility patterns in control, regeneration and other tissues and stages. The model does not reflect a real situation, in that enhancers are not necessarily located in the genomic distribution depicted. TF: transcription factor; COF: co-factor; Pol-II: RNA polymerase II; CR: chromatin remodelers; DRREs: damage-responsive regulatory elements; L3: third instar larva.

C
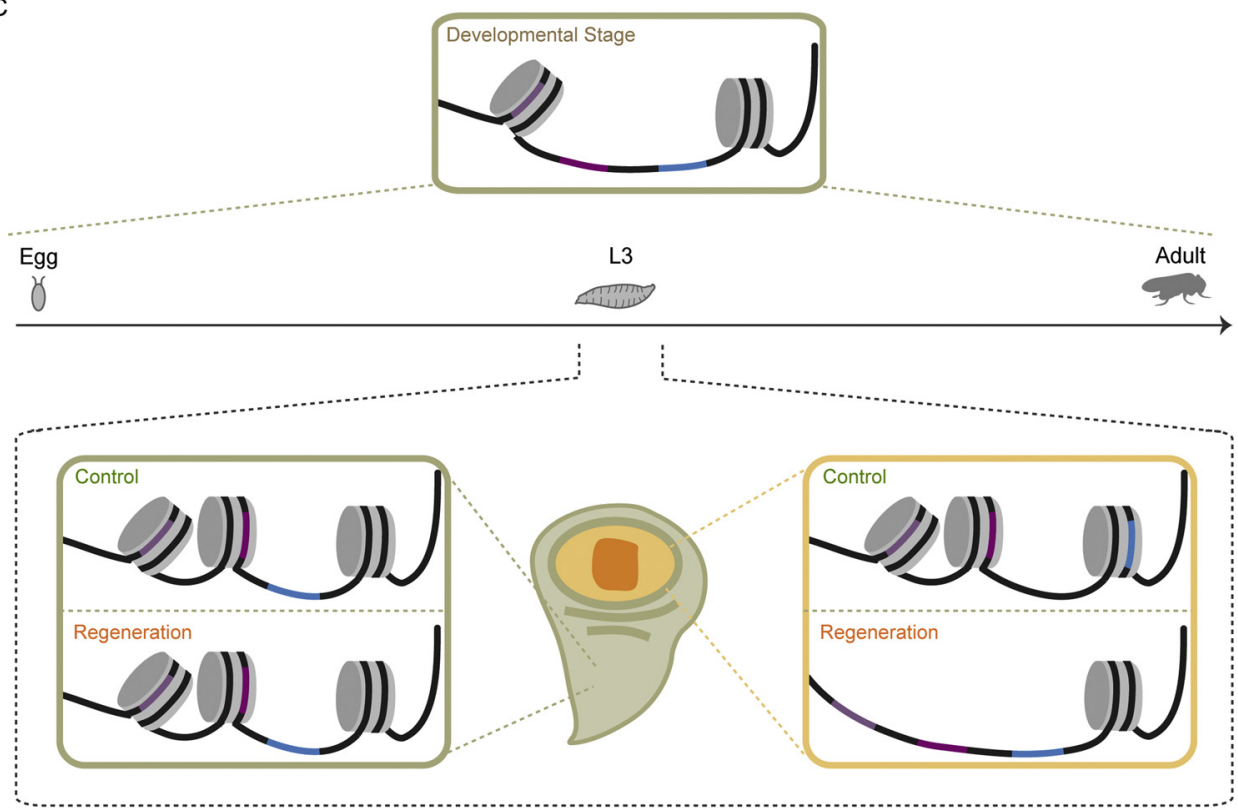

IDRRE

reused-eDRRE

novel-eDRRE

a correlation between histone modifications and states; however, there is no mark or combination that perfectly matches any one state. For example, around $40 \%$ of Drosophila embryonic enhancers lack H3K27ac, yet they are active [101]. Moreover, there is no evidence that such marks are either sufficient or necessary for transcription. One recent study in Drosophila has demonstrated that correlation does not imply causation; and that it is not in fact the mark (H3K4me1) that is required for transcription, but the histone methyltransferase governing that mark [102,103]. Additionally, it has been demonstrated that transcription can occur in the absence of histone marks in promoters of regulated genes in Drosophila [104].

Transcription factors bound to enhancer sequences also provide information on enhancer activity. Often, each enhancer is bound to a specific transcription factor or to a particular combination of them [105]. Together with the search for universal rules for enhancer activation, other properties have been studied. Enhancers possess some inherent promoter capacity and can recruit Pol-II and transcription factors [106] leading to the transcription of enhancer-RNA (eRNA) [107-109]. In a recent study, it was demonstrated that the degree of enhancer or promoter activity is reflected in the level and directionality of eRNA transcription in the fruit fly [110].

Finally, one of the most fascinating properties of enhancers is their functional autonomy: their capacity to retain transcription-activating functions outside their endogenous loci ([111]; reviewed in [88,94].
Taking advantage of this feature and by using reporter lines, enhancer activation has been described in cells involved in regeneration in both zebrafish and Drosophila [71,75,78].

\subsection{Enhancer accessibility during regeneration}

Different types of regulatory elements have recently been identified based on their accessibility in zebrafish heart (TREEs) and Drosophila wing disc regeneration, using high- throughput genome-wide analyses such as chromatin profiling and ATAC-seq [75,112]. In the case of fruit flies, three main types of damage-responsive regulatory elements (DRREs) have been defined [75] (Fig. 2B). The first, named increasing DRREs (iDRREs), are found in chromatin regions that are already accessible in the wild-type tissue under study, but become more accessible during regeneration, indicating fine-tuning of gene expression. The damage enhancer found at the WNT locus in regeneration of the wing disc [71] is included in this class. Although it may seem that these enhancers are already active before regeneration occurs, analysis using reporter lines has shown that compared to their basal activation pattern, some iDRREs are indeed ectopically activated in the wound [75].

The second type of DRREs have been named emerging DRREs (eDRREs) because they correspond to open regions only detected after damage [75]. Some eDRREs can be further classified into reused eDRREs, which are co-opted from other developmental stages or tissues 
and reused in regeneration and novel eDRREs, which act exclusively in the damaged tissue. Reused eDRREs account for around 50\% of the enhancers thought to be specific to wing disc regeneration [75]. As mentioned, during regeneration, cells have to undergo respecification and proliferate to replace lost tissue; in this manner, the tissue recapitulates some necessary developmental traits. Reactivation of two embryonic enhancers in the epicardial cell layer of zebrafish and mouse hearts explains how some epicardial genes that are transcriptionally activated during embryonic development can be re-induced after injury [113]. Similarly, an enhancer that triggers Bone morphogenetic protein 5 (Bmp5) expression during mouse skeletal development is also used in bone repair. Interestingly, the same enhancer is sufficient to trigger gene expression in mesenchymal and epithelial cells in multiple tissues, suggesting that it might contain an injury-responsive enhancer element [114].

Novel eDRREs account for almost 50\%, approximately, of the enhancers thought to be specific of wing disc regeneration [75]. This last category could, in theory, represent unique regeneration enhancers. The leptin $B$ enhancer found in zebrafish regeneration seems to be this type of enhancer: it has been proved that it plays a crucial role in regeneration, but it does not seem to be required at all, either during development or in basal heart homeostasis [78]. However, further comparative analysis with more tissues and stages is needed to allow us to refine our knowledge of the occurrence of the different enhancer types.

\subsection{Post-translational chromatin modifications at regeneration enhancers}

Chromatin immunoprecipitation followed by sequencing (ChIP-seq) has been used to map globally the chromatin state of various cis-regulatory elements at different times after acute muscle trauma and the total number of enhancer elements has been determined based on the presence of H3K4me1 and H3K27ac [73]. Similarly, ChIP-seq experiments on such histone marks have demonstrated that $90 \%$ and $50 \%$ of DRREs in zebrafish heart and wing disc respectively, are marked during regeneration [75,112]. Intriguingly, despite some DRREs not being marked by any of the common modifications, they are still activated upon damage [75]. On the other hand, the need to lose repressive modifications seems to be crucial for activation $[66,67,69,70]$.

\subsection{Transcription factors: the link between signaling and chromatin}

Accumulating evidence from many model systems indicates that the combinatorial interplay of multiple transcription factors, each with its own partially overlapping temporal window of expression, is a prominent regulator of context-specific binding. A recent study has identified four transcription factors involved in the de novo epithelialization of skin ulcers in mice [115]. Moreover, some studies indicate that there could be global regulatory rules, represented as codes for motif composition that ultimately determine enhancer activity (reviewed in [116]. Such codes are simultaneously regulated by the transcription factors found at each time in the cell. Hence, variations in this spectrum will generate different target gene expression profiles in different cells. Thus, transcription factors can induce alterations and enable patterning through development in the same cell type (reviewed in $[105,116]$ ). The same kind of logic is postulated for regeneration, where a plethora of these proteins are specifically activated upon damage, leading to a variation of the preexistent spectrum. As mentioned above, regeneration does not depend only on the genome sequence but on genome activity and cell physiology. Cell physiology, represented as pro-regeneration signals, governs the activation of the set of specific transcription factors that help to modulate genome activity through the recognition of their binding sites, written as codes of motif composition in enhancers.

Motif analysis has enabled to identify the binding sites required in zebrafish heart and fly wing disc regeneration. RUNX2B, STAT1, RFX2, SPI1 and NFBKB are the transcription factors whose binding sites are the most enriched within the enhancers required in zebrafish heart regeneration; whereas STAT92E, Trl, Fd68A and Grh motifs are those most enriched within Drosophila's wing disc regeneration enhancers [75,112]. Moreover, the recent comparison of transcriptomic data obtained from three different organs in three different species (mouse liver, zebrafish heart and Drosophila wing disc) has resulted in identifying a set of 21 transcription factors conserved and upregulated in all three of them $[75,112,117]$. Interestingly, some of these transcription factors (STAT92E, Sd, and Myb) present a motif enriched in DRREs.

\section{Conclusions and future challenges}

Drosophila is currently shedding some light into the basic and conserved mechanisms behind the regenerative process, although it is still not clear if imaginal discs can regenerate in the wild under adverse conditions. Comparative studies between model organisms should provide valuable information to help us understand how transcription associated with regeneration is regulated.

An increase in transcription that correlates with several changes associated to chromatin dynamics has been observed after damage in the wing imaginal disc. These change include: increased chromatin accessibility, increase in the expression levels of specific transcription factors and formation of new chromatin loops, and loss of repressive chromatin modifications (Fig. 3).

New approaches should provide more insight into the how changes in chromatin dynamics contribute to changes in cell plasticity during the regeneration process: 1 ) Using high-throughput techniques, such as 4C and High-C conformation capture, that allow us to interrogate the 3D structure of the genome. The information this could provide would be important to map the contacts between different chromosomal regions, such as those between enhancers and promoters; 2) Genome

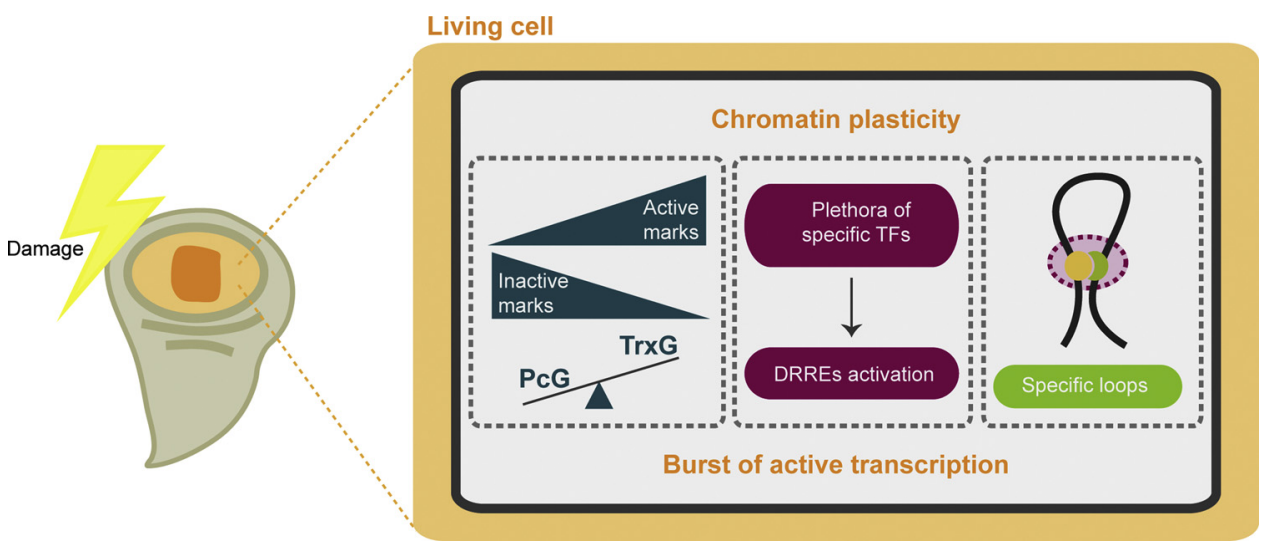

Fig. 3. Changes in transcription and chromatin plasticity after damage. An increase in transcription in cells responding to damage correlates with several changes associated to chromatin dynamics and increase of cell plasticity. These changes include: loss of repressive chromatin modifications, increase in the expression levels of specific transcription factors and formation of new chromatin loops. 
editing, using the CRISPR/Cas9 system or similar ones, is needed to validate the requirement for regeneration-associated enhancers as well as to demonstrate that their removal affects transcription; the same CRISPR technology may be a useful tool to modify enhancer architecture or to study the role of specific histone modifications at enhancers. 3) Determining the contribution of the non-coding genome to regeneration. Information on such transcripts is already available for the systems on which RNA-seq has been performed. Focusing on transcripts that are induced during regeneration and producing the appropriate mutations should provide instrumental information regarding their potential contribution to the process.

\section{Acknowledgments}

We thank J. Esteban-Collado for his help in manuscript edition. Authors are funded by the Institució Catalana de Recerca i Estudis Avançats (Icrea Academia award) to MC, grants BFU2012-36888 and BFU2015- 67623-P to FS and MC, Ministerio de Economía y Competitividad, Spain. CK acknowledges funding from the Ministerio de Economía y Competitividad, Spain to the EMBL partnership, and the support of the CERCA Institute / Generalitat de Catalunya and Centro de Excelencia Severo Ochoa. Parts of this review are from the PhD thesis by Elena Vizcaya-Molina, University of Barcelona, 2018.

\section{References}

[1] S.E. Iismaa, X. Kaidonis, A.M. Nicks, N. Bogush, K. Kikuchi, N. Naqvi, R.P. Harvey, A. Husain, R.M. Graham, Comparative regenerative mechanisms across different mammalian tissues, Npj Regen. Med. 3 (2018), https://doi.org/10.1038/s41536018-0044-5.

[2] T.H. Morgan, Regeneration, (1901) https://archive.org/details/ regeneration00morggoog.

[3] K.D. Poss, L.G. Wilson, M.T. Keating, Heart regeneration in zebrafish, Science (80-.) 298 (2002) 2188-2190, https://doi.org/10.1126/science.1077857.

[4] S.G. Cummings, H.R. Bode, Head regeneration and polarity reversal inHydra attenuata can occur in the absence of DNA synthesis, Wilhelm Roux's Arch. Dev. Biol. 194 (1984) 79-86, https://doi.org/10.1007/BF00848347.

[5] A.E. Bely, K.G. Nyberg, Evolution of animal regeneration: re-emergence of a field, Trends Ecol. Evol. (Amst.) 25 (2010) 161-170, https://doi.org/10.1016/j.tree. 2009.08.005.

[6] J.M. Slack, Animal regeneration: ancestral character or evolutionary novelty? EMBO Rep. 18 (2017) 1497-1508, https://doi.org/10.15252/embr.201643795.

[7] A.W. Seifert, J.R. Monaghan, M.D. Smith, B. Pasch, A.C. Stier, F. Michonneau, M. Maden, The influence of fundamental traits on mechanisms controlling appendage regeneration, Biol. Rev. 87 (2012) 330-345, https://doi.org/10.1111/j. 1469-185X.2011.00199.x.

[8] M.H. Yun, Changes in regenerative capacity through lifespan, Int. J. Mol. Sci. 16 (2015) 25392-25432, https://doi.org/10.3390/ijms161025392.

[9] A. Zhao, H. Qin, X. Fu, What determines the regenerative capacity in animals? Bioscience 66 (2016) 735-746, https://doi.org/10.1093/biosci/biw079.

[10] A. Sánchez Alvarado, P.A. Tsonis, Bridging the regeneration gap: genetic insights from diverse animal models, Nat. Rev. Genet. 7 (2006) 873-884, https://doi.org/ $10.1038 / \mathrm{nrg} 1923$.

[11] E.M. Tanaka, P.W. Reddien, The cellular basis for animal regeneration, Dev. Cell 21 (2011) 172-185, https://doi.org/10.1016/j.devcel.2011.06.016.

[12] C.-H. Chen, K.D. Poss, Regeneration genetics, Annu. Rev. Genet. 51 (2017), https://doi.org/10.1146/annurev-genet-120116-024554 annurev-genet-120116024554.

[13] S.-Y. Liu, C. Selck, B. Friedrich, R. Lutz, M. Vila-Farré, A. Dahl, H. Brandl, N. Lakshmanaperumal, I. Henry, J.C. Rink, Reactivating head regrowth in a regeneration-deficient planarian species, Nature 500 (2013) 81-84, https://doi.org/ 10.1038/nature12414.

[14] Y. Umesono, J. Tasaki, Y. Nishimura, M. Hrouda, E. Kawaguchi, S. Yazawa, O. Nishimura, K. Hosoda, T. Inoue, K. Agata, The molecular logic for planarian regeneration along the anterior-posterior axis, Nature 500 (2013) 73-76, https:// doi.org/10.1038/nature12359.

[15] A.W. Seifert, S.G. Kiama, M.G. Seifert, J.R. Goheen, T.M. Palmer, M. Maden, Skin shedding and tissue regeneration in African spiny mice (Acomys), Nature 489 (2012) 561-565, https://doi.org/10.1038/nature11499.

[16] E.R. Porrello, A.I. Mahmoud, E. Simpson, J.A. Hill, J.A. Richardson, E.N. Olson, H.A. Sadek, Transient regenerative potential of the neonatal mouse heart, Science (80-.) 331 (2011) 1078-1080, https://doi.org/10.1126/science.1200708.

[17] K.M. Bullard, M.T. Longaker, H.P. Lorenz, Fetal wound healing: current biology, World J. Surg. 27 (2003) 54-61, https://doi.org/10.1007/s00268-002-6737-2.

[18] S.-J. Shieh, T.-C. Cheng, Regeneration and repair of human digits and limbs: fact and fiction, Regeneration 2 (2015) 149-168, https://doi.org/10.1002/reg2.41.

[19] J.S. Jaszczak, A. Halme, Arrested development: coordinating regeneration with development and growth in Drosophila melanogaster, Curr. Opin. Genet. Dev. 40
(2016) 87-94, https://doi.org/10.1016/j.gde.2016.06.008.

[20] I.K. Hariharan, F. Serras, Imaginal disc regeneration takes flight, Curr. Opin. Cell Biol. 48 (2017) 10-16, https://doi.org/10.1016/j.ceb.2017.03.005.

[21] J.P. Brockes, A. Kumar, Comparative aspects of animal regeneration, Annu. Rev. Cell Dev. Biol. 24 (2008) 525-549, https://doi.org/10.1146/annurev.cellbio.24. 110707.175336

[22] M.I. Worley, L. Setiawan, I.K. Hariharan, Regeneration and transdetermination in Drosophila imaginal discs, Annu. Rev. Genet. 46 (2012) 289-310, https://doi.org/ 10.1146/annurev-genet-110711-155637.

[23] J.V. Beira, R. Paro, The legacy of Drosophila imaginal discs, Chromosoma. 125 (2016) 573-592, https://doi.org/10.1007/s00412-016-0595-4.

[24] C. Bergantiños, X. Vilana, M. Corominas, F. Serras, Imaginal discs: renaissance of a model for regenerative biology, BioEssays 32 (2010) 207-217, https://doi.org/10. 1002/bies.200900105.

[25] M. Levin, Bioelectric mechanisms in regeneration: unique aspects and future perspectives, Semin. Cell Dev. Biol. 20 (2009) 543-556, https://doi.org/10.1016/ j.semcdb.2009.04.013.

[26] W. Razzell, I.R. Evans, P. Martin, W. Wood, Calcium flashes orchestrate the wound inflammatory response through DUOX activation and hydrogen peroxide release, Curr. Biol. 23 (2013) 424-429, https://doi.org/10.1016/j.cub.2013.01.058.

[27] S.K. Yoo, C.M. Freisinger, D.C. LeBert, A. Huttenlocher, Early redox, Src family kinase, and calcium signaling integrate wound responses and tissue regeneration in zebrafish, J. Cell Biol. 199 (2012) 225-234, https://doi.org/10.1083/jcb. 201203154.

[28] P. Niethammer, C. Grabher, A.T. Look, T.J. Mitchison, A tissue-scale gradient of hydrogen peroxide mediates rapid wound detection in zebrafish, Nature. 459 (2009) 996-999, https://doi.org/10.1038/nature08119.

[29] P. Santabárbara-Ruiz, M. López-Santillán, I. Martínez-Rodríguez, A. Binagui-Casas, L. Pérez, M. Milán, M. Corominas, F. Serras, ROS-induced JNK and p38 signaling is required for unpaired cytokine activation during Drosophila regeneration, PLoS Genet. (2015), https://doi.org/10.1371/journal.pgen.1005595.

[30] S. Restrepo, K. Basler, Drosophila wing imaginal discs respond to mechanical injury via slow InsP3R-mediated intercellular calcium waves, Nat. Commun. 7 (2016) 12450, https://doi.org/10.1038/ncomms12450.

[31] P. Santabárbara-Ruiz, J. Esteban-Collado, L. Pérez, G. Viola, J.F. Abril, M. Milán, M. Corominas, F. Serras, Ask1 and Akt act synergistically to promote ROS-dependent regeneration in Drosophila, PLoS Genet. 15 (2019) e1007926, , https:// doi.org/10.1371/journal.pgen.1007926.

[32] D. Burzyn, W. Kuswanto, D. Kolodin, J.L. Shadrach, M. Cerletti, Y. Jang, E. Sefik, T.G. Tan, A.J. Wagers, C. Benoist, D. Mathis, A special population of regulatory T cells potentiates muscle repair, Cell 155 (2013) 1282-1295, https://doi.org/10. 1016/j.cell.2013.10.054.

[33] T.A. Petrie, N.S. Strand, C. Tsung-Yang, J.S. Rabinowitz, R.T. Moon, R.T. Moon, Macrophages modulate adult zebrafish tail fin regeneration, Development. 141 (2014) 2581-2591, https://doi.org/10.1242/dev.098459.

[34] T.A. Wynn, K.M. Vannella, Macrophages in tissue repair, regeneration, and fibrosis, Immunity 44 (2016) 450-462, https://doi.org/10.1016/j.immuni.2016.02. 015.

[35] C.E. Fogarty, N. Diwanji, J.L. Lindblad, M. Tare, A. Amcheslavsky, K. Makhijani, K. Brückner, Y. Fan, A. Bergmann, Extracellular reactive oxygen species drive apoptosis-induced proliferation via Drosophila macrophages, Curr. Biol. 26 (2016) 575-584, https://doi.org/10.1016/j.cub.2015.12.064.

[36] S.J. Khan, S.N.F. Abidi, A. Skinner, Y. Tian, R.K. Smith-Bolton, The Drosophila Duox maturation factor is a key component of a positive feedback loop that sustains regeneration signaling, PLoS Genet. 13 (2017) e1006937, , https://doi.org/ 10.1371/journal.pgen.1006937.

[37] A.R. Brock, M. Seto, R.K. Smith-Bolton, Cap-N-Collar Promotes Tissue Regeneration by 206 (2017), pp. 1505-1520, https://doi.org/10.1534/genetics. 116.196832/-/DC1.1.

[38] M. Bosch, F. Serras, E. Martín-Blanco, J. Baguñà, JNK signaling pathway required for wound healing in regenerating Drosophila wing imaginal discs, Dev. Biol. 280 (2005) 73-86, https://doi.org/10.1016/j.ydbio.2005.01.002.

[39] C. Bergantiños, M. Corominas, F. Serras, Cell death-induced regeneration in wing imaginal discs requires JNK signalling, Development 137 (2010) 1169-1179, https://doi.org/10.1242/dev.045559.

[40] A. Repiso, C. Bergantinos, F. Serras, Cell fate respecification and cell division orientation drive intercalary regeneration in Drosophila wing discs, Development 140 (2013) 3541-3551, https://doi.org/10.1242/dev.095760.

[41] G. Sun, K.D. Irvine, Regulation of Hippo signaling by Jun kinase signaling during compensatory cell proliferation and regeneration, and in neoplastic tumors, Dev. Biol. 350 (2011) 139-151, https://doi.org/10.1016/j.ydbio.2010.11.036.

[42] S. Vriz, S. Reiter, B. Galliot, Cell death, A Program to Regenerate., Curr. Top. Dev. Biol. (2014), https://doi.org/10.1016/B978-0-12-391498-9.00002-4.

[43] T. Katsuyama, F. Comoglio, M. Seimiya, E. Cabuy, R. Paro, During Drosophila disc regeneration, JAK/STAT coordinates cell proliferation with Dilp8-mediated developmental delay, Proc. Natl. Acad. Sci. U. S. A 112 (2015), https://doi.org/10. 1073/pnas.1423074112 E2327-36.

[44] J. López-Luque, D. Caballero-Díaz, A. Martinez-Palacián, C. Roncero, J. MorenoCàceres, M. García-Bravo, E. Grueso, A. Fernández, E. Crosas-Molist, M. GarcíaÁlvaro, A. Addante, E. Bertran, A.M. Valverde, Á. González-Rodríguez, B. Herrera, L. Montoliu, T. Serrano, J.-C. Segovia, M. Fernández, E. Ramos, A. Sánchez, I. Fabregat, Dissecting the role of epidermal growth factor receptor catalytic activity during liver regeneration and hepatocarcinogenesis, Hepatology 63 (2016) 604-619, https://doi.org/10.1002/hep.28134.

[45] G. Sun, K.D. Irvine, Control of growth during regeneration, Curr. Top. Dev. Biol. 108 (2014) 95-120, https://doi.org/10.1016/B978-0-12-391498-9.00003-6. 
[46] A. Paksa, J. Rajagopal, The epigenetic basis of cellular plasticity, Curr. Opin. Cell Biol. 49 (2017) 116-122, https://doi.org/10.1016/j.ceb.2018.01.003.

[47] T. Yadav, J.P. Quivy, G. Almouzni, Chromatin plasticity: A versatile landscape that underlies cell fate and identity, Science 361 (2018) 1332-1336, https://doi.org/ $10.1126 /$ science.aat8950.

[48] J. Baguñà, Planarian regeneration between 1960s and 1990s: from skilful baffled ancestors to bold integrative descendants, A personal account, Semin. Cell Dev. Biol. (2018), https://doi.org/10.1016/j.semcdb.2018.04.011.

[49] P.W. Reddien, The cellular and molecular basis for planarian regeneration, Cell 175 (2018) 327-345, https://doi.org/10.1016/j.cell.2018.09.021.

[50] J. Baguñà, The planarian neoblast: the rambling history of its origin and some current black boxes, Int. J. Dev. Biol. 56 (2012) 19-37, https://doi.org/10.1387/ ijdb.113463jb.

[51] J.J. Henry, P.A. Tsonis, Molecular and cellular aspects of amphibian lens regeneration, Prog. Retin. Eye Res. 29 (2010) 543-555, https://doi.org/10.1016/j. preteyeres.2010.07.002.

[52] S. Siebert, F. Anton-Erxleben, T.C.G. Bosch, Cell type complexity in the basal metazoan Hydra is maintained by both stem cell based mechanisms and transdifferentiation, Dev. Biol. 313 (2008) 13-24, https://doi.org/10.1016/j.ydbio. 2007.09.007.

[53] B. Galliot, Hydra, a fruitful model system for 270 years, Int. J. Dev. Biol. 56 (2012) 411-423, https://doi.org/10.1387/ijdb.120086bg.

[54] C. Jopling, E. Sleep, M. Raya, M. Martí, A. Raya, J.C.I. Belmonte, Zebrafish heart regeneration occurs by cardiomyocyte dedifferentiation and proliferation, Nature 464 (2010) 606-609, https://doi.org/10.1038/nature08899.

[55] H. Sánchez-Iranzo, M. Galardi-Castilla, C. Minguillón, A. Sanz-Morejón, J.M. González-Rosa, A. Felker, A. Ernst, G. Guzmán-Martínez, C. Mosimann, N. Mercader, Tbx5a lineage tracing shows cardiomyocyte plasticity during zebrafish heart regeneration, Nat. Commun. 9 (2018) 428, https://doi.org/10.1038/ s41467-017-02650-6.

[56] J. López-Luque, I. Fabregat, Revisiting the liver: from development to regeneration - what we ought to know!, Int. J. Dev. Biol. 62 (2018) 441-451, https://doi.org/ $10.1387 / \mathrm{ijdb} .170264 \mathrm{JL}$

[57] S. Schwartz, C. Rhiner, Reservoirs for repair? Damage-responsive stem cells and adult tissue regeneration in drosophila, Int. J. Dev. Biol. 62 (2018) 465-471, https://doi.org/10.1387/ijdb.180056cr.

[58] R.K. Smith-Bolton, M.I. Worley, H. Kanda, I.K. Hariharan, Regenerative Growth in Drosophila Imaginal Discs Is Regulated by Wingless and Myc, Dev. Cell 16 (2009) 797-809, https://doi.org/10.1016/j.devcel.2009.04.015.

[59] S.C. Herrera, G. Morata, Transgressions of compartment boundaries and cell reprogramming during regeneration in Drosophila, Elife 3 (2014) e01831, , https:// doi.org/10.7554/eLife.01831.

[60] C. Maurange, N. Lee, R. Paro, Signaling meets chromatin during tissue regeneration in Drosophila, Curr. Opin. Genet. Dev. 16 (2006) 485-489, https://doi.org/ 10.1016/j.gde.2006.08.007

[61] E. HADORN, Problems of determination and transdetermination, Brookhaven Symp. Biol. (1965) 148-161.

[62] L. Maves, G. Schubiger, Transdetermination in Drosophila imaginal discs: a model for understanding pluripotency and selector gene maintenance, Curr. Opin. Genet. Dev. 13 (2003) 472-479, https://doi.org/10.1016/j.gde.2003.08.006.

[63] G. Morata, S.C. Herrera, Cell reprogramming during regeneration in Drosophila: transgression of compartment boundaries, Curr. Opin. Genet. Dev. 40 (2016) 11-16, https://doi.org/10.1016/j.gde.2016.05.008.

[64] T. Katsuyama, R. Paro, Epigenetic reprogramming during tissue regeneration, FEBS Lett. 585 (2011) 1617-1624, https://doi.org/10.1016/j.febslet.2011.05. 010 .

[65] B. Schuettengruber, H.-M. Bourbon, L. Di Croce, G. Cavalli, Genome regulation by Polycomb and trithorax: 70 years and counting, Cell 171 (2017) 34-57, https:// doi.org/10.1016/j.cell.2017.08.002.

[66] T. Shaw, P. Martin, Epigenetic reprogramming during wound healing: loss of polycomb-mediated silencing may enable upregulation of repair genes, EMBO Rep. 10 (2009) 881-886, https://doi.org/10.1038/embor.2009.102.

[67] N. Lee, C. Maurange, L. Ringrose, R. Paro, Suppression of Polycomb group proteins by JNK signalling induces transdetermination in Drosophila imaginal discs, Nature 438 (2005) 234-237, https://doi.org/10.1038/nature04120.

[68] A. Skinner, S.J. Khan, R.K. Smith-Bolton, Trithorax regulates systemic signaling during Drosophila imaginal disc regeneration, Development 142 (2015) 3500-3511, https://doi.org/10.1242/dev.122564.

[69] K.J. Schuster, R.K. Smith-Bolton, Taranis protects regenerating tissue from fate changes induced by the wound response in Drosophila, Dev. Cell 34 (2015) 119-128, https://doi.org/10.1016/j.devcel.2015.04.017.

[70] S. Stewart, Z.-Y. Tsun, J.C.I. Belmonte, A histone demethylase is necessary for regeneration in zebrafish, Proc. Natl. Acad. Sci. 106 (2009) 19889-19894, https:// doi.org/10.1073/pnas.0904132106.

[71] R.E. Harris, L. Setiawan, J. Saul, I.K. Hariharan, Localized epigenetic silencing of a damage-activated WNT enhancer limits regeneration in mature Drosophila imaginal discs, Elife 5 (2016), https://doi.org/10.7554/eLife.11588.

[72] K. Sousounis, M. Looso, N. Maki, C.J. Ivester, T. Braun, P.A. Tsonis, Transcriptome analysis of newt Lens regeneration reveals distinct gradients in gene expression patterns, PLoS One 8 (2013) e61445, , https://doi.org/10.1371/journal.pone. 0061445.

[73] C.A. Aguilar, R. Pop, A. Shcherbina, A. Watts, R.W. Matheny, D. Cacchiarelli, W.M. Han, E. Shin, S.A. Nakhai, Y.C. Jang, C.T. Carrigan, C.A. Gifford, M.A. Kottke, M. Cesana, J. Lee, M.L. Urso, A. Meissner, Transcriptional and chromatin dynamics of muscle regeneration after severe trauma, Stem Cell Reports 7 (2016) 983-997, https://doi.org/10.1016/j.stemcr.2016.09.009.
[74] S. Rodius, G. Androsova, L. Götz, R. Liechti, I. Crespo, S. Merz, P.V. Nazarov, N. de Klein, C. Jeanty, J.M. González-Rosa, A. Muller, F. Bernardin, S.P. Niclou, L. Vallar, N. Mercader, M. Ibberson, I. Xenarios, F. Azuaje, Analysis of the dynamic co-expression network of heart regeneration in the zebrafish, Sci. Rep. 6 (2016) 26822, https://doi.org/10.1038/srep26822.

[75] E. Vizcaya-Molina, C.C. Klein, F. Serras, R.K. Mishra, R. Guigó, M. Corominas, Damage-responsive elements in Drosophila regeneration, Genome Res. 28 (2018) 1852-1866, https://doi.org/10.1101/gr.233098.117.

[76] K. Yang, J. Kang, Tissue regeneration enhancer elements: a way to unlock endogenous healing power, Dev. Dyn. (2019) 34-42, https://doi.org/10.1002/dvdy. 24676.

[77] M. Schubiger, A. Sustar, G. Schubiger, Regeneration and transdetermination: the role of wingless and its regulation, Dev. Biol. 347 (2010) 315-324, https://doi. org/10.1016/j.ydbio.2010.08.034.

[78] J. Kang, J. Hu, R. Karra, A.L. Dickson, V.A. Tornini, G. Nachtrab, M. Gemberling, J.A. Goldman, B.L. Black, K.D. Poss, Modulation of tissue repair by regeneration enhancer elements, Nature 532 (2016) 201-206, https://doi.org/10.1038/ nature17644.

[79] A.R. Gehrke, E. Neverett, Y.-J. Luo, A. Brandt, L. Ricci, R.E. Hulett, A. Gompers, J.G. Ruby, D.S. Rokhsar, P.W. Reddien, M. Srivastava, Acoel genome reveals the regulatory landscape of whole-body regeneration, Science (80-.) 363 (2019), https://doi.org/10.1126/science.aau6173 eaau6173.

[80] J. Dekker, M.A. Marti-Renom, L.A. Mirny, Exploring the three-dimensional organization of genomes: interpreting chromatin interaction data, Nat. Rev. Genet. 14 (2013) 390-403, https://doi.org/10.1038/nrg3454.

[81] M.J. Rowley, V.G. Corces, The three-dimensional genome: principles and roles of long-distance interactions, Curr. Opin. Cell Biol. 40 (2016) 8-14, https://doi.org/ 10.1016/j.ceb.2016.01.009.

[82] Y.B. Schwartz, G. Cavalli, Three-dimensional genome organization and function in Drosophila, Genetics 205 (2017) 5-24, https://doi.org/10.1534/genetics.115 185132.

[83] C. Cubeñas-Potts, M.J. Rowley, X. Lyu, G. Li, E.P. Lei, V.G. Corces, Different enhancer classes in Drosophila bind distinct architectural proteins and mediate unique chromatin interactions and 3D architecture, Nucleic Acids Res. 45 (2017) 1714-1730, https://doi.org/10.1093/nar/gkw1114.

[84] B. Bonev, G. Cavalli, Organization and function of the 3D genome, Nat. Rev. Genet. 17 (2016) 661-678, https://doi.org/10.1038/nrg.2016.112.

[85] T. Sexton, G. Cavalli, The role of chromosome domains in shaping the functional genome, Cell 160 (2015) 1049-1059, https://doi.org/10.1016/j.cell.2015.02.040.

[86] M.A. Zabidi, C.D. Arnold, K. Schernhuber, M. Pagani, M. Rath, O. Frank, A. Stark Enhancer-core-promoter specificity separates developmental and housekeeping gene regulation, Nature 518 (2015) 556-559, https://doi.org/10.1038/ nature13994.

[87] N. Koenecke, J. Johnston, B. Gaertner, M. Natarajan, J. Zeitlinger, Genome-wide identification of Drosophila dorso-ventral enhancers by differential histone acetylation analysis, Genome Biol. 17 (2016) 196, https://doi.org/10.1186/s13059016-1057-2.

[88] R.R. Catarino, A. Stark, Assessing sufficiency and necessity of enhancer activities for gene expression and the mechanisms of transcription activation, Genes Dev. 32 (2018) 202-223, https://doi.org/10.1101/gad.310367.117.

[89] V. Charoensawan, S.C. Janga, M.L. Bulyk, M.M. Babu, S.A. Teichmann, DNA sequence preferences of transcriptional activators correlate more strongly than repressors with nucleosomes, Mol. Cell 47 (2012) 183-192, https://doi.org/10. 1016/j.molcel.2012.06.028.

[90] I. Barozzi, M. Simonatto, S. Bonifacio, L. Yang, R. Rohs, S. Ghisletti, G. Natoli, Coregulation of transcription factor binding and nucleosome occupancy through DNA features of mammalian enhancers, Mol. Cell 54 (2014) 844-857, https://doi. org/10.1016/j.molcel.2014.04.006.

[91] A.P. Boyle, S. Davis, H.P. Shulha, P. Meltzer, E.H. Margulies, Z. Weng, T.S. Furey, G.E. Crawford, High-resolution mapping and characterization of open chromatin across the genome, Cell 132 (2008) 311-322, https://doi.org/10.1016/j.cell. 2007.12.014.

[92] A. Rada-Iglesias, R. Bajpai, T. Swigut, S.A. Brugmann, R.A. Flynn, J. Wysocka, A unique chromatin signature uncovers early developmental enhancers in humans, Nature 470 (2011) 279-283, https://doi.org/10.1038/nature09692.

[93] E. Calo, J. Wysocka, Modification of enhancer chromatin: what, how, and why? Mol. Cell 49 (2013) 825-837, https://doi.org/10.1016/j.molcel.2013.01.038.

[94] D. Shlyueva, G. Stampfel, A. Stark, Transcriptional enhancers: from properties to genome-wide predictions, Nat. Rev. Genet. 15 (2014) 272-286, https://doi.org/ $10.1038 / \mathrm{nrg} 3682$.

[95] H.K. Long, S.L. Prescott, J. Wysocka, Ever-changing landscapes: transcriptional enhancers in development and evolution, Cell 167 (2016) 1170-1187, https://doi org/10.1016/j.cell.2016.09.018.

[96] L. Skalska, R. Stojnic, J. Li, B. Fischer, G. Cerda-Moya, H. Sakai, S. Tajbakhsh, S. Russell, B. Adryan, S.J. Bray, Chromatin signatures at Notch-regulated enhancers reveal large-scale changes in H3K56ac upon activation, EMBO J. 34 (2015) 1889-1904, https://doi.org/10.15252/embj.201489923.

[97] J.A. Simon, R.E. Kingston, Mechanisms of Polycomb gene silencing: knowns and unknowns, Nat. Rev. Mol. Cell Biol. 10 (2009) 697-708, https://doi.org/10.1038/ nrm2763.

[98] A.H.F.M. Peters, J.E. Mermoud, D. O’Carroll, M. Pagani, D. Schweizer, N. Brockdorff, T. Jenuwein, Histone H3 lysine 9 methylation is an epigenetic imprint of facultative heterochromatin, Nat. Genet. 30 (2001) 77-80, https://doi org/10.1038/ng789.

[99] B.E. Bernstein, T.S. Mikkelsen, X. Xie, M. Kamal, D.J. Huebert, J. Cuff, B. Fry, A. Meissner, M. Wernig, K. Plath, R. Jaenisch, A. Wagschal, R. Feil, S.L. Schreiber, 
E.S. Lander, A bivalent chromatin structure marks key developmental genes in embryonic stem cells, Cell 125 (2006) 315-326, https://doi.org/10.1016/j.cell. 2006.02.041.

[100] R. Ostuni, V. Piccolo, I. Barozzi, S. Polletti, A. Termanini, S. Bonifacio, A. Curina, E. Prosperini, S. Ghisletti, G. Natoli, Latent Enhancers Activated by Stimulation in Differentiated Cells, Cell 152 (2013) 157-171, https://doi.org/10.1016/j.cell 2012.12.018.

[101] S. Bonn, R.P. Zinzen, C. Girardot, E.H. Gustafson, A. Perez-Gonzalez, N. Delhomme, Y. Ghavi-Helm, B. Wilczyński, A. Riddell, E.E.M. Furlong, Tissuespecific analysis of chromatin state identifies temporal signatures of enhancer activity during embryonic development, Nat. Genet. 44 (2012) 148-156, https:// doi.org/10.1038/ng.1064.

[102] K.M. Dorighi, T. Swigut, T. Henriques, N.V. Bhanu, B.S. Scruggs, N. Nady, C.D. Still, B.A. Garcia, K. Adelman, J. Wysocka, Mll3 and Mll4 facilitate enhancer RNA synthesis and transcription from promoters independently of H3K4 monomethylation, Mol. Cell 66 (2017) 568-576, https://doi.org/10.1016/j.molcel. 2017.04.018 e4.

[103] R. Rickels, H.-M. Herz, C.C. Sze, K. Cao, M.A. Morgan, C.K. Collings, M. Gause, Y. Takahashi, L. Wang, E.J. Rendleman, S.A. Marshall, A. Krueger, E.T. Bartom, A. Piunti, E.R. Smith, N.A. Abshiru, N.L. Kelleher, D. Dorsett, A. Shilatifard, Histone H3K4 monomethylation catalyzed by Trr and mammalian COMPASS-like proteins at enhancers is dispensable for development and viability, Nat. Genet. 49 (2017) 1647-1653, https://doi.org/10.1038/ng.3965.

[104] S. Pérez-Lluch, E. Blanco, H. Tilgner, J. Curado, M. Ruiz-Romero, M. Corominas, R. Guigó, Absence of canonical marks of active chromatin in developmentally regulated genes, Nat. Genet. 47 (2015) 1158-1167, https://doi.org/10.1038/ng. 3381.

[105] M.A. Zabidi, A. Stark, Regulatory enhancer-Core-Promoter communication via transcription factors and cofactors, Trends Genet. 32 (2016) 801-814, https://doi. org/10.1016/j.tig.2016.10.003.

[106] F. Koch, R. Fenouil, M. Gut, P. Cauchy, T.K. Albert, J. Zacarias-Cabeza, S. Spicuglia, A.L. de la Chapelle, M. Heidemann, C. Hintermair, D. Eick, I. Gut, P. Ferrier, J.-C. Andrau, Transcription initiation platforms and GTF recruitment at tissue-specific enhancers and promoters, Nat. Struct. Mol. Biol. 18 (2011) 956-963, https://doi.org/10.1038/nsmb.2085.

[107] D. Tuan, S. Kong, K. Hu, Transcription of the hypersensitive site HS2 enhancer in erythroid cells, Proc. Natl. Acad. Sci. U. S. A 89 (1992) 11219-11223 (accessed September 1, 2018), http://www.ncbi.nlm.nih.gov/pubmed/1454801.

[108] F. De Santa, I. Barozzi, F. Mietton, S. Ghisletti, S. Polletti, B.K. Tusi, H. Muller, J. Ragoussis, C.-L. Wei, G. Natoli, A large fraction of extragenic RNA pol II transcription sites overlap enhancers, PLoS Biol. 8 (2010) e1000384, , https://doi. org/10.1371/journal.pbio.1000384.

[109] M.T.Y. Lam, H. Cho, H.P. Lesch, D. Gosselin, S. Heinz, Y. Tanaka-Oishi, C. Benner, M.U. Kaikkonen, A.S. Kim, M. Kosaka, C.Y. Lee, A. Watt, T.R. Grossman, M.G. Rosenfeld, R.M. Evans, C.K. Glass, Rev-Erbs repress macrophage gene expression by inhibiting enhancer-directed transcription, Nature 498 (2013) 511-515, https://doi.org/10.1038/nature12209.

[110] O. Mikhaylichenko, V. Bondarenko, D. Harnett, I.E. Schor, M. Males, R.R. Viales, E.E.M. Furlong, The degree of enhancer or promoter activity is reflected by the levels and directionality of eRNA transcription, Genes Dev. 32 (2018) 42-57, https://doi.org/10.1101/gad.308619.117.

[111] J. Banerji, S. Rusconi, W. Schaffner, Expression of a beta-globin gene is enhanced by remote SV40 DNA sequences, Cell 27 (1981) 299-308 (Accessed 01, September 2018), http://www.ncbi.nlm.nih.gov/pubmed/6277502.

[112] J.A. Goldman, G. Kuzu, N. Lee, J. Karasik, M. Gemberling, M.J. Foglia, R. Karra, A.L. Dickson, F. Sun, M.Y. Tolstorukov, K.D. Poss, Resolving heart regeneration by replacement histone profiling, Dev. Cell 40 (2017) 392-404, https://doi.org/10. 1016/j.devcel.2017.01.013 e5.

[113] G.N. Huang, J.E. Thatcher, J. McAnally, Y. Kong, X. Qi, W. Tan, J.M. DiMaio, J.F. Amatruda, R.D. Gerard, J.A. Hill, R. Bassel-Duby, E.N. Olson, C/EBP transcription factors mediate epicardial activation during heart development and injury, Science 338 (2012) 1599-1603, https://doi.org/10.1126/science.1229765.

[114] C.A. Guenther, Z. Wang, E. Li, M.C. Tran, C.Y. Logan, R. Nusse, L. Pantalena-Filho, G.P. Yang, D.M. Kingsley, A distinct regulatory region of the Bmp5 locus activates gene expression following adult bone fracture or soft tissue injury, Bone 77 (2015) 31-41, https://doi.org/10.1016/j.bone.2015.04.010.

[115] M. Kurita, T. Araoka, T. Hishida, D.D. O’Keefe, Y. Takahashi, A. Sakamoto, M. Sakurai, K. Suzuki, J. Wu, M. Yamamoto, R. Hernandez-Benitez, A. Ocampo, P. Reddy, M.N. Shokhirev, P. Magistretti, E. Núñez Delicado, H. Eto, K. Harii, J.C. Izpisua Belmonte, In vivo reprogramming of wound-resident cells generates skin epithelial tissue, Nature 561 (2018) 243-247, https://doi.org/10.1038/ s41586-018-0477-4.

[116] F. Spitz, E.E.M. Furlong, Transcription factors: from enhancer binding to developmental control, Nat. Rev. Genet. 13 (2012) 613-626, https://doi.org/10.1038/ nrg3207.

[117] X. Sun, J.-C. Chuang, M. Kanchwala, L. Wu, C. Celen, L. Li, H. Liang, S. Zhang, T. Maples, L.H. Nguyen, S.C. Wang, R.A.J. Signer, M. Sorouri, I. Nassour, X. Liu, J. Xu, M. Wu, Y. Zhao, Y.-C. Kuo, Z. Wang, C. Xing, H. Zhu, Suppression of the SWI/SNF component Arid1a promotes mammalian regeneration, Cell Stem Cell 18 (2016) 456-466, https://doi.org/10.1016/j.stem.2016.03.001. 